$\mathrm{N}$, Engelbrecht $\mathrm{V}$ et al. Cystic leukoencephalopathy without megalencephaly: a distinct disease entity in 15 children. Neurology April (2 of 2) 2005;64:1411-1416). (Reprints: Dr Jutta Gartner, Department of Pediatrics and Pediatric Neurology, Georg August University, Faculty of Medicine, Robert-Koch-Strasse 40, 37075 Gottingen, Germany).

COMMENT. The authors distinguish this autosomal recessive disease from a previously described cystic leukoencephalopathy with megalencephaly (MLC), caused by a mutation of MLC1 gene. (Van der Knapp et al, 1995; Progress in Pediatric Neurology III, 1997;p557). In MLC, the MRI showed supratentorial white matter swelling and subcortical cysts, which contrasted with a mild clinical course.

\title{
CEREBRAL PALSY AND NEONATAL ENCEPHALOPATHY
}

The type and severity of cerebral palsy (CP) and pattern of associated disability in children with or without preceding neonatal encephalopathy (NE) were compared in a population-based case-control study of patients followed for 6 years at the Children's Hospital, Westmead, Sydney, Australia. Of 276 infants with NE, $25(9.1 \%)$ died in the neonatal period, and of the 251 neonatal NE survivors, $32(13 \%)$ developed CP by 5 years of age. Of term infants with CP, $24 \%$ followed NE. CP following NE was more likely in males, more severe, spastic quadriplegic in type, and more commonly complicated by cognitive and speech impairment, epilepsy, severe disability, and death by 6 years. (Badawi N, Felix JF, Kurinczuk JJ et al. Cerebral palsy following newborn encephalopathy: a population-based study. Dev Med Child Neurol May 2005;47:293-298). (Respond: Nadia Badawi PhD FRACP, Department of Neonatology, Children's Hospital at Westmead, Locked Bag 4001, Westmead, NSW 2145, Australia).

COMMENT. Term infants with $\mathrm{CP}$ and NE have a poorer prognosis compared to those without NE. One out of every five will die in the first 5 years of life. In a commentary by Dr Karin B Nelson, National Institutes of Health, USA (Dev Med Child Neurol 2005;47:292), the importance of causal factors in NE was stressed, a subject addressed by the authors in a previous study (Badawi N et al. BMJ 1998;317:1549-1553 and 1554-1558).

\section{SPASTIC DIPLEGIC AND TETRAPLEGIC CEREBRAL PALSY COMPARED}

Risk factors of cerebral palsy (CP), seizures, CP severity, EEG, and MRI findings were compared in 38 children with spastic diplegic (DCP) and 48 with spastic tetraplegic (TCP), in a report from Medical University of Bialystok, Poland. The Apgar score was lower in TCP cases than DCP, the gross motor function was more limited, mental retardation more frequent, cerebral atrophy on MRI more frequent ( $31 \%$ cf $5 \%$ ), epilepsy more common ( $50 \%$ cf $16 \%$ ) and more often intractable. Periventricular leukomalacia on MRI was more frequent in DCP $(76 \%)$ than in TCP $(44 \%)$. Gestational history was not related to increased risk of DCP or TCP; the frequencies of cesarean section, low birth weight, and perinatal pathology were the same in both groups. (Kulak W, Sobaniec W, Smigielska-Kuzia J et al. Pediatr Neurol May 2005;32:311-317). (Respond: Dr Kulak, Department of Pediatric Neurology an 
d Rehabilitation, Medical University of Bialystok, ul Waszyngtona 17, 15-274 Bialystok, Poland).

COMMENT. Spastic diplegia involves the legs more than the arms, and is frequently associated with premature birth. Spastic quadriplegia, the most severe form of $\mathrm{CP}$, affects all 4 limbs, but impairment of motor function is usually more severe in the upper limbs. In the classification of CP by Crothers and Paine (1959), 65\% of cases were spastic (19\% quadriplegic, $2.8 \%$ diplegic, $40.5 \%$ hemiplegic), $22 \%$ extrapyramidal, and $13 \%$ mixed types. Changes in the classification of $\mathrm{CP}$ in the years 1954-90 were related to increased survival rates of preterm infants (Hagberg B et al. Acta Paediatr Suppl 1996;416:48-52). Bilateral spastic forms (diplegias, quadriplegias, and extrapyramidal) were most prevalent, and $75 \%$ were in preterm and $45 \%$ in term infants. In the above Polish study (Kulak et al), $55 \%$ of both DCP and TCP cases were born prematurely. In the above Australian study (Badawi et al), the classification of $\mathrm{CP}$ in term infants (\% of total cases; and with/without encephalopathy) was as follows: spastic quadriplegic $(17 \% ; 31 / 12 \%)$, spastic diplegic $(23 \% ; 19 / 24 \%)$, hemiplegic $(34 \% ; 22 / 38 \%)$, athetotic dystonic $(17 \% ; 25 / 14 \%)$, and ataxic hypotonic $(10 \% ; 3 / 11 \%)$.

\section{CONGENITAL MALFORMATIONS}

\section{GENETIC VARIETIES OF JOUBERTS SYNDROME}

Four families with linkage to two loci for Jouberts syndrome (JS), JBTS1 or JBTS2, and clinical and radiographic correlations for 4 known genetic causes of JS-related disorders (JSRD) are described in a report from University of California-San Diego, La Jolla, CA, and centers in Europe and the Middle East. The clinical manifestations of JS are hypotonia, ataxia, mental retardation, oculomotor apraxia, and dysregulation of neonatal breathing. The molar tooth sign (MTS) is the diagnostic neuroradiological feature of JS, a complex midbrain-hindbrain malformation consisting of cerebellar vermis hypoplasia, deep interpeduncular fossa, and thickened, elongated, maloriented superior cerebellar peduncles. JSRD are characterized by the association of the MTS with abnormalities of other organs, including retinal dystrophy and polycystic and fibrotic kidneys, optic coloboma, polydactyly, liver fibrosis, and other CNS malformations (eg polymicrogyria). JBTS1 and -3 features are restricted to the CNS, whereas JBTS2 involves multiple organs, including kidney, retina, and liver as well as CNS. (Valente EM, Marsh SE, Castori M et al. Distinguishing the four genetic causes of Jouberts syndrome-related disorders. Ann Neurol April 2005;57:513-519). (Respond: Dr Gleeson, University of California-San Diego, Leichtag 332, 9500 Gilman Drive, La Jolla, CA 92093).

COMMENT. In addition to Jouberts, syndromes of cerebellar vermis agenesis or hypoplasia include Dandy-Walker malformation, Walker-Warburg (lissencephaly, retinal abnormalities and hydrocephalus), Meckel-Gruber (occipital encephalocele, polycystic kidneys, poydactyly and hydrocephalus), and atypical Dandy-Walker with facial angioma. Some cases of vermian agenesis are associated with urinary excretion of succinyl-purines and pipecolic acid (Bordarier C, Aicardi J. Dev Med Child Neurol 1990;32:285-294; See Progress in Pedeiatric Neurology I, 1991;312-313). 